\title{
Strabismus: Wandel in Diagnostik und Therapie
}

\section{H. Thaller-Antlanger}

Dieser Bericht ist ein ganz persönlicher Rückblick auf eine 33-jährige strabologische Tätigkeit. Aus der Vielfalt der Themen werden die Diagnostik und Therapie der Amblyopie, der Korrespondenz und des Binokularsehens, des Strabismus divergens intermittens, des Strabismus sursoadductorius mit Binokularfunktionen und der Trochlearisparese herausgenommen.

Standen bei der Amblyopietherapie vor 30 Jahren wegen der viel zu späten Erfassung der Patienten die apparative Pleoptik mit dem Euthyskop ( 2 bis $3 \times 4$ Wochen stationäre Aufnahme!), bei Säuglingen und Kleinkindern die in der Hand des Unerfahrenen nicht ungefährliche Atropinkur, gefolgt von strenger fazialer Okklusion über Jahre im Vordergrund, so liegt heute der Schwerpunkt auf der Früherfassung und Prophylaxe durch Teilzeitokklusion. Selbstverständlich werden Kinder mit exzentrischer Fixation nach wie vor streng okkludiert und lange nachbetreut sowie ebenfalls apparativ behandelt, jedoch gehört die zeitaufwändige ,ungeliebte“ Euthyskopbehandlung der Vergangenheit an.

Ferner haben wir zur Kenntnis genommen, dass der Aufwand einer Korrespondenzbehandlung (Prismenüberkorrektur über lange Zeit, Operation in die Divergenz) mit anschlieBender intensiver Binokularschulung in keiner Relation zum erwünschten Erfolg stand. Die Korrespondenz ist abhängig vom Schielbeginn, von der Schielform und vom Zeitpunkt des Einsetzens der Therapie.

Eine wesentliche Voraussetzung für das Binokularsehen ist $\mathrm{m}$. E. neben der Behandlung mit Prismen die bestmögliche operative Stellungskorrektur im Gebrauchsblickfeld. Auch müssen wir spontane Funktionsentwicklungen zulassen (Ausnahme normosensorisches konvergentes Spätschielen).

Beim Strabismus divergens intermittens (Pseudodivergenzexzesstyp) wird heute die konservative Therapie des

\section{Wenn es kreist, ist es gut!}

\section{Mojon}

Abt. für Strabologie und Neuroophthalmologie, KSSG St. Gallen, Schweiz

Das dynamische Stereosehen wird klinisch zu selten geprüft. Am einfachsten kann es durch Testung des Pulfrich-Phänomens untersucht werden. Beim spontanen Pulfrich-Phänomen scheint dem Patienten, ohne dass ein Graufilter vor ein Auge gehalten werden muss, ein in einer frontoparallelen Ebene sich bewegendes Pendel sich elliptisch zu bewegen. Diverse Erkrankungen können ein spontanes Pulfrich-Phänomen auslösen: Optikusneuropathien, vor allem die Optikusneuritis, Makulopathien, Anisometropien, Strabismus, Medientrübungen, zentrale Sehstörungen und stärkere Anisokorien. Auch wenn wie genannt ein Strabismus ein spontanes Phänomen auslösen kann, ist dies sehr selten. Wichtiger scheint hingegen für die Strabologie das induzierbare Pulfrich-Phänomen manifesten Fernwinkels mit Antisuppressionsübungen, Trainieren der willkürlichen und akkommodativen Konvergenz vermieden, da daran eine wirksame spätere operative Behandlung scheitern kann. Auch die vor 30 Jahren übliche präoperative Vorbereitung mit einer alternierenden Brillenokklusion oder dem alternierenden Tragen von Folienprismen durch 3 Monate ist heute der vom Strabismus divergens intermittens bevorzugten Altersgruppe (Schulkinder, Erwachsene) nicht mehr zuzumuten.

Aufklärung über Rezidive und mögliche Zweiteingriffe, sowie der Hinweis auf den Verlust eines bestehenden Panoramasehens stehen heute im Vordergrund.

Abschließend wird auf die vor allem im angloamerikanischen Schrifttum noch immer nicht vollzogene Differenzierung zwischen einem kongenitalen Strabismus sursoadductorius und der neurogen erworbenen Trochlearisparese hingewiesen und auf die differentialdiagnostisch richtungsweisenden Parameter eingegangen.

Beim Blick in die Zukunft scheint die Strabologie vor allem an den Kliniken zunehmend funktionelle Aufgaben gegenüber der vorwiegend morphologisch orientierten Ophthalmologie übernehmen zu müssen.

In das Spezialfach hineindrängende Randgebiete wie Neuroophthalmologie (bereits fest etabliert), Neurophysiologie, Immunologie, Innere Medizin, Labordiagnostik, Psychologie und Bildgebung erfordern intensive Ausbildung und Weiterbildung auch in fachfremden Disziplinen.

Es wird auch in Zukunft Änderungen geben - nicht ändern sollte sich die Zuwendung zum Patienten, die klinische Untersuchung und Diagnosestellung, die Zuhilfenahme apparativer Diagnostik soviel wie nötig, so wenig wie möglich (Wirtschaftlichkeit) und der persönliche ärztliche Einsatz.

zu sein, bei dem der Patient das Phänomen erst nach Vorhalten eines Graufilters vor ein Auge sieht. Es zeigt sich, dass bei Patienten, die nur einen positiven Trefftest aufweisen, ohne dass die gängigen Stereotests erkannt werden, meist ein Pulfrich-Phänomen induziert werden kann. Wenn höhere Stereofunktionen bestehen, ist praktisch immer ein Pulfrich-Phänomen $\mathrm{zu}$ induzieren. Strabologisch am wichtigsten scheinen die Fälle bei denen sogar der Trefftest negativ ist und nur ein induzierbares Pulfrich-Phänomen besteht. Dieser alleinige Nachweis dieses Phänomens kann dann den funktionellen Nutzen eines Prismenausgleiches oder einer Schieloperation aufzeigen. 